\title{
Declarações de Nascidos Vivos: uma avaliação da qualidade das informações em dois municípios da Região Metropolitana de João Pessoa, Paraíba, Brasil
}

\author{
Certificates of Live Birth: an evaluation of the quality of \\ information in two municipalities in the metropolitan region of \\ João Pessoa, state of Paraíba, Brazil
}

\author{
Dixis Figueroa Pedraza' (1) \\ 'Departamento de Enfermagem, Universidade Estadual da Paraíba (UEPB) - Campina Grande (PB), Brasil.
}

Como citar: Figueroa Pedraza D. Declarações de Nascidos Vivos: uma avaliação da qualidade das informações em dois municípios da Região Metropolitana de João Pessoa, Paraíba, Brasil. Cad Saúde Colet, 2021;29(2):271-278. https://doi. org/10.1590/1414-462X202129020107

\begin{abstract}
Resumo
Introdução: As informações sobre estatísticas vitais ressaltam-se por sua importância como subsídios para ações adequadas em diversos setores, tornando relevante avaliar a qualidade delas. Objetivo: Descrever a completude e a concordância de variáveis selecionadas da Declaração de Nascidos Vivos (DNV) de crianças hospitalizadas de dois municípios da Região Metropolitana de João Pessoa, com base nos prontuários hospitalares. Método: Realizou-se um estudo descritivo considerando menores de 1 ano residentes nos municípios de Cabedelo e Bayeux, nascidos em 2013, hospitalizados no mesmo ano em hospitais públicos pediátricos do estado da Paraíba. Com base nos dados dos prontuários hospitalares, as DNVs foram analisadas em relação à completude e à concordância, segundo o coeficiente Kappa (variáveis categóricas) ou o coeficiente de correlação intraclasse (variáveis quantitativas). Resultados: Na avaliação das DNVs, a idade gestacional destacou-se por apresentar completude muito ruim e concordância apenas razoável. A quantidade de filhos mortos também foi classificada em concordância razoável. Os problemas na qualidade da informação dessas variáveis inviabilizam seu uso em estudos epidemiológicos e como subsídio para as políticas públicas. Conclusão: A completude e a concordância das variáveis analisadas denotaram boa qualidade no preenchimento das DNVs, exceto para a idade gestacional e o número de filhos mortos. Palavras-chave: nascidos vivos; sistemas de informação; estatísticas vitais.
\end{abstract}

\begin{abstract}
Background: Data on vital statistics stand out for their importance in supporting appropriate actions in various sectors; therefore, assessment of their quality is relevant. Objective: To describe the completeness and concordance of selected variables of the Certificates Live Birth (CLB) of hospitalized children in two municipalities of the metropolitan region of João Pessoa based on hospital records. Method: A descriptive study was carried out considering children under one year of age living in the municipalities of Cabedelo and Bayeux, born in 2013, hospitalized in the same year in public pediatric hospitals in the state of Paraíba. Based on data from hospital records, CLB were analyzed in relation to their completeness and agreement using the Kappa Coefficient (categorical variables) or the Intraclass Correlation Coefficient (quantitative variables). Results: In the evaluation of the CLB, gestational age stood out for presenting
\end{abstract}

Este é um artigo publicado em acesso aberto (Open Access) sob a licença Creative Commons Attribution, que permite uso, distribuição e reprodução em qualquer meio, sem restrições desde que o trabalho original seja corretamente citado.

\footnotetext{
Trabalho realizado em hospitais públicos com maternidade do Estado da Paraíba - Bayeux, Cabedelo (PB), Brasil. Correspondência: Dixis Figueroa Pedraza. E-mail: dixisfigueroa@gmail.com

Fonte de financiamento: nenhuma.

Conflito de interesses: nada a declarar.

Recebido em: Mar. 12, 2019. Aprovado em: Mar. 10, 2020
} 
very poor completeness and only reasonable agreement. The number of dead children was also rated with reasonable agreement. The problems in the quality of information on these variables prevent their use in epidemiological studies and as a support to public policies. Conclusion: The completeness and agreement of the analyzed variables showed good quality in the completion of the CLB, except for gestational age and number of dead children.

Keywords: live births; information systems; vital statistics.

\section{INTRODUÇÃO}

No Brasil, o Sistema de Informações sobre Nascidos Vivos (SINASC), alimentado por meio da Declaração de Nascido Vivo (DNV), representa uma ferramenta essencial para o funcionamento dos serviços de saúde, o controle social no âmbito do Sistema Único de Saúde (SUS) e o desenvolvimento de pesquisas na área materno-infantil ${ }^{1,2}$. Temas como a descrição do perfil de nascidos vivos, a identificação de fatores de risco para desfechos infantis e a avaliação dos serviços e programas de saúde têm sido objeto de estudo por meio das informações do SINASC'.

A consolidação do SINASC enquanto fonte de dados de grande relevância para a saúde pública exige esforços relacionados à qualidade das suas informações como condição necessária para a análise objetiva da situação sanitária e da necessidade do direcionamento das políticas públicas ${ }^{2-4}$. Essas circunstâncias tornam imprescindível a avaliação da qualidade das informações do sistema nas diferentes regiões do país ${ }^{2,3}$. Nesse sentido, revisões da literatura têm constatado diferenças regionais na qualidade das informações ${ }^{2,3,5}$, ressaltando-se a importância de estudos em áreas específicas como forma de propor soluções adaptadas a cada realidade ${ }^{5,6}$.

A despeito de os resultados dos estudos sobre a qualidade do SINASC indicarem boa completude e confiabilidade nos dados registrados para a maioria das informações, inconsistências têm sido destacadas no preenchimento das variáveis ocupação, escolaridade materna, raça/cor, número de consultas de pré-natal, idade gestacional e número de filhos mortos $^{2,4,5,7}$. No entanto, esses resultados podem não representar a realidade do estado da Paraíba, tendo em vista que ele carece de publicações sobre a temática ${ }^{2,3,5}$. Além disso, são poucos os estudos sobre a qualidade do SINASC que abordam especificamente situações infantis de risco ${ }^{2,5,8}$ e que podem se relacionar ao preenchimento das DNVs, cujas informações são vitais para a análise da saúde materno-infantil em grupos de maior vulnerabilidade ${ }^{8}$.

Considerando ainda que a descentralização do SINASC para as maternidades locais é defendida com base na sua importância para a consolidação e análise dos dados presentes nas DNVs, de forma a intervir com maior rapidez nas situações de risco do recém-nascido ${ }^{9}$, este estudo teve por objetivo descrever a completude e a concordância de variáveis selecionadas das DNVs de crianças hospitalizadas de dois municípios da Região Metropolitana de João Pessoa conforme os prontuários hospitalares.

\section{MÉTODO}

Realizou-se um estudo descritivo com base na análise da completude e da concordância de variáveis das DNVs, referentes a menores de 1 ano residentes em Cabedelo e Bayeux, Paraíba, nascidos em 2013. Incluíram-se as crianças que necessitaram de internação no mesmo ano em algum dos quatro hospitais públicos pediátricos do estado da Paraíba com serviço de hospitalização pediátrica para habitantes nesses municípios.

Os municípios do estudo foram escolhidos considerando suas semelhanças em posição geográfica (localização na região metropolitana da capital do estado com acesso à rede de serviços pactuados), grau de urbanização, indicadores demográfico-sociais, recursos econômicos e tradição na organização de serviços primários de saúde (cobertura populacional da Estratégia Saúde da Família de 100\%). Cabedelo possui um sistema de saúde composto por 28 equipes da Estratégia Saúde da Família, e Bayeux, por 19. Em 2012, Cabedelo possuía uma população de 60.226 habitantes, e Bayeux, de 100.543. No ano seguinte, foram registrados no SINASC 965 nascimentos em Cabedelo e 1.395 em Bayeux.

Os dados foram coletados entre 12 e 21 de março de 2014 nos quatro hospitais públicos do Estado que internam crianças residentes nos municípios do estudo, sendo que um está 
localizado no município de Cabedelo, e três, no município de João Pessoa. O Serviço de Arquivo Médico e Estatística de cada instituição viabilizou a consulta aos prontuários de todas as crianças nascidas em 2013, procedendo-se à identificação das residentes nos municípios de interesse, que representaram os sujeitos do estudo $(\mathrm{N}=278)$. Esse total corresponde a $11,8 \%$ dos nascidos vivos de Cabedelo e Bayeux em 2013 (http://svs.aids.gov.br/dantps/centrais-de-conteudos/ paineis-de-monitoramento/natalidade/nascidos-vivos/). A equipe de campo foi formada por profissionais e estudantes da área de saúde, com experiência prévia em trabalho de campo. O controle de qualidade do estudo incluiu: treinamento e padronização dos entrevistadores, construção de manual de instruções, realização de estudo-piloto e supervisão da coleta de dados.

A coleta de dados ocorreu mediante o preenchimento de formulário em branco idêntico à DNV original por acadêmicos treinados para a pesquisa, com informações dos prontuários das mães (na área de obstetrícia) e de seus respectivos filhos nos arquivos hospitalares: estado civil da mãe, escolaridade materna, tipo de gravidez, tipo de parto, sexo da criança, idade materna, quantidade de filhos vivos, quantidade de filhos mortos, idade gestacional, peso ao nascer, Apgar no $1^{\circ}$ minuto, Apgar no $5^{\circ}$ minuto e número de consultas de pré-natal. O preenchimento foi feito segundo as indicações do Manual de Instruções para o Preenchimento da Declaração de Nascido Vivo. Além disso, obteve-se cópia fiel da DNV no órgão de processamento do município.

Os dados coletados foram organizados em planilhas eletrônicas e digitados em dupla entrada. O aplicativo Validate do software Epi Info, versão 6.04b, foi usado para analisar a consistência dos dados, gerando o banco final que foi utilizado nas análises estatísticas.

Avaliou-se a completude das variáveis das DNVs e dos prontuários. A completude diz respeito aos campos com informação completa ${ }^{10}$. Conforme proposto por Romero e Cunha desde 2007 , os casos com menos de $5 \%$ de dados não preenchidos foram considerados como completude excelente; de $5 \%$ a $10 \%$ de não preenchimento, bom; de $10 \%$ a $20 \%$, regular; de $20 \%$ a $50 \%$, ruim; e acima de $50 \%$, muito ruim ${ }^{10}$.

Para a avaliação de concordância das variáveis entre as DNVs e os prontuários, excluíram-se todos os registros não preenchidos. A concordância refere-se à consistência das informações registradas em um instrumento quando comparadas às de outra ferramenta ${ }^{2}$. Para avaliar a concordância, existem diversos testes estatísticos, cujo uso depende das variáveis em análise. Quando a variável de interesse é dicotômica e a estimativa ocorre por dois avaliadores ou dois instrumentos, sugere-se o uso da estatística Kappa de Cohen. Para a análise no caso de variável ordinal, utiliza-se, classicamente, da estatística Kappa com pesos quadráticos. Quando as variáveis de interesse são quantitativas, emprega-se comumente o coeficiente de correlação intraclasse, que pode ser calculado por algoritmos diversos, como o aleatório de uma via (os avaliadores são diferentes), o aleatório de duas vias (os avaliadores são os mesmos e escolhidos aleatoriamente) e o misto de duas vias (os avaliadores são os únicos possíveis) ${ }^{11}$.

Dessa forma, no caso das variáveis categóricas (estado civil da mãe, escolaridade da mãe, tipo de gravidez, tipo de parto e sexo da criança), utilizou-se do coeficiente Kappa. Para a variável categórica ordinal escolaridade materna, empregou-se o coeficiente Kappa ponderado com pesos quadráticos. Para a medida de concordância das variáveis quantitativas (idade da mãe, quantidade de filhos nascidos vivos e mortos, idade gestacional, peso ao nascer, Apgar no $1^{\circ}$ e $5^{\circ}$ minutos e número de consultas de pré-natal), utilizou-se do coeficiente de correlação intraclasse, adotando-se o modelo two way mixed effect mode ${ }^{12}$. O cálculos foram realizadas com o software Stata, versão 12.0. Os valores entre 0,80 e 1,00 foram considerados como concordância quase perfeita; entre 0,60 e 0,79, substancial; entre 0,40 e 0,59, moderada; entre 0,20 e 0,39, razoável; e abaixo de 0,20, pobre 2,13 .

A pesquisa foi aprovada pelo Comitê de Ética em Pesquisa da Universidade Estadual da Paraíba, com o protocolo de número 19689613.3.0000.5187. A participação na pesquisa foi condicionada à assinatura do Termo de Consentimento Livre e Esclarecido. 


\section{RESULTADOS}

Nas DNVs analisadas, constatou-se um alto percentual de completude, maior do que o encontrado para o caso dos prontuários na maioria das variáveis. A idade gestacional foi a variável com menor completude nas DNVs $(42,45 \%)$, classificada como muito ruim, sendo a quantidade de registros completos nos prontuários com um percentual superior $(85,61 \%)$, caracterizando preenchimento regular. O Apgar no $1^{\circ} \mathrm{e}$ o Apgar no $5^{\circ}$ minuto também apresentaram maior completude nos prontuários, entretanto, em ambos os instrumentos, o preenchimento foi caracterizado como excelente. Enquanto a completude das variáveis estado civil da mãe, escolaridade materna, quantidade de filhos vivos e quantidade de filhos mortos das DNVs situou-se na categoria excelente, a classificação dos prontuários foi regular para a primeira, ruim para a segunda e muito ruim para as outras duas. Para o número de consultas de pré-natal, as DNVs apresentaram completude boa, e os prontuários, completude ruim (Tabela 1).

Segundo o índice Kappa, a concordância foi quase perfeita $(\geq 0,80)$ para as variáveis tipo de gravidez, tipo de parto e sexo da criança. Concordâncias similares aferidas pelo ICC foram identificadas para idade materna, peso ao nascer, Apgar no $1^{\circ} \mathrm{e} 5^{\circ}$ minutos e número de consultas de pré-natal. As menores concordâncias, classificadas como razoáveis, foram encontradas para idade gestacional (ICC $=0,29$ ) e quantidade de filhos mortos (ICC $=0,28$ ) (Tabela 2).

\section{DISCUSSÃO}

O presente trabalho buscou descrever a completude e a concordância de variáveis contidas nas DNVs de crianças hospitalizadas residentes em dois municípios do estado da Paraíba. A qualidade observada para a maioria das variáveis vai ao encontro da tendência apontada na cidade de Recife ${ }^{14}$ e no município do Rio de Janeiro, no qual os dados coletados se referiam a crianças de risco clínico potencialmente elevado ${ }^{8}$. A qualidade das informações do SINASC

Tabela 1. Completude de variáveis selecionadas nas Declarações de Nascidos Vivos (DNVs) e nos prontuários de crianças menores de 1 ano hospitalizadas em quatro hospitais públicos pediátricos da Paraíba, Brasil. Bayeux e Cabedelo, $2013(\mathrm{~N}=278)$

\begin{tabular}{|c|c|c|c|c|}
\hline \multirow{2}{*}{ Variáveis } & \multicolumn{2}{|c|}{ Registros (n) } & \multicolumn{2}{|c|}{ Completude (\%) } \\
\hline & DNV & Prontuário & DNV & Prontuário \\
\hline Estado civil da mãe & 266 & 235 & 95,68 & 84,53 \\
\hline Escolaridade materna & 273 & 195 & 98,20 & 70,14 \\
\hline Tipo de gravidez & 278 & 272 & 100 & 97,84 \\
\hline Tipo de parto & 276 & 274 & 99,28 & 98,56 \\
\hline Sexo da criança & 277 & 263 & 99,64 & 94,60 \\
\hline Idade materna & 274 & 273 & 98,56 & 98,20 \\
\hline Quantidade de filhos vivos & 266 & 139 & 95,68 & 50,00 \\
\hline Quantidade de filhos mortos & 269 & 108 & 96,76 & 38,85 \\
\hline Idade gestacional & 118 & 238 & 42,45 & 85,61 \\
\hline Peso ao nascer & 276 & 272 & 99,28 & 97,84 \\
\hline Apgar no $1^{\circ}$ minuto & 271 & 274 & 97,48 & 98,56 \\
\hline Apgar no $5^{\circ}$ minuto & 271 & 274 & 97,48 & 98,56 \\
\hline Número de consultas de pré-natal & 257 & 175 & 92,45 & 62,95 \\
\hline
\end{tabular}


Tabela 2. Concordância nas informações de variáveis selecionadas das Declarações de Nascidos Vivos (DNVs) e dos prontuários de crianças menores de 1 ano hospitalizadas em quatro hospitais públicos pediátricos da Paraíba, Brasil. Bayeux e Cabedelo, 2013 (N = 278)

\begin{tabular}{|c|c|c|c|c|}
\hline \multirow{2}{*}{ Variáveis categóricas } & \multirow{2}{*}{$\begin{array}{l}\text { Registros } \\
\text { (n) }\end{array}$} & \multirow{2}{*}{$\begin{array}{c}\text { Concordância } \\
\text { (\%) }\end{array}$} & \multicolumn{2}{|c|}{ Kappa (IC95\%) } \\
\hline & & & Simples & Ponderado \\
\hline Estado civil da mãe & 225 & 80,95 & $0,75(0,72-0,79)$ & - \\
\hline Escolaridade materna & 190 & 73,16 & $0,59(0,55-0,67)$ & $\begin{array}{l}0,55(0,51- \\
0,60)\end{array}$ \\
\hline Tipo de gravidez & 272 & 99,98 & $0,99(0,96-1,00)$ & - \\
\hline Tipo de parto & 272 & 98,90 & $0,97(0,77-0,98)$ & - \\
\hline Sexo da criança & & 98,48 & $0,96(0,93-0,98)$ & - \\
\hline Variáveis numéricas & $\begin{array}{l}\text { Registros } \\
\text { (n) }\end{array}$ & $\begin{array}{c}\text { Concordância } \\
\text { (\%) }\end{array}$ & \multicolumn{2}{|c|}{ ICC (IC95\%) } \\
\hline Idade materna & 269 & 81,41 & \multicolumn{2}{|c|}{$0,81(0,79-0,90)$} \\
\hline Quantidade de filhos vivos & 140 & 78,99 & \multicolumn{2}{|c|}{$0,72(0,62-0,83)$} \\
\hline Quantidade de filhos mortos & 104 & 84,62 & \multicolumn{2}{|c|}{$0,28(0,12-0,31)$} \\
\hline Idade gestacional & 105 & 64,89 & \multicolumn{2}{|c|}{$0,29(0,13-0,39)$} \\
\hline Peso ao nascer & 270 & 93,70 & \multicolumn{2}{|c|}{$0,94(0,91-0,99)$} \\
\hline Apgar no $1^{\circ}$ minuto & 269 & 97,40 & \multicolumn{2}{|c|}{$0,96(0,95-0,99)$} \\
\hline Apgar no $5^{\circ}$ minuto & 269 & 96,28 & \multicolumn{2}{|c|}{$0,92(0,90-0,94)$} \\
\hline $\begin{array}{l}\text { Número de consultas de } \\
\text { pré-natal }\end{array}$ & 164 & 82,32 & \multicolumn{2}{|c|}{$0,80(0,76-0,89)$} \\
\hline
\end{tabular}

IC95\%: Intervalo de Confiança de 95\%

ICC: coeficiente de correlação intraclasse

também foi confirmada recentemente por meio dos resultados com coleta de dados primários no estudo Nascer no Brasil ${ }^{15}$. Dessa forma, é possível atestar o potencial da DNV e do SINASC como fonte de informação confiável sobre os nascidos vivos no Brasil, possibilitando análises como a elaboração de diagnósticos de saúde, a vigilância e o monitoramento de recém-nascidos e a avaliação de ações de saúde materno-infantis ${ }^{4,16}$.

Conforme observado no presente estudo, avaliações do SINASC, tanto de abrangência nacional/regional $\left.\right|^{7,17,18}$ quanto local $\left.\right|^{8,19,20}$, destacaram o preenchimento incompleto da idade gestacional. Informações ignoradas ou em branco são decorrentes de vários fatores, tais como dificuldades de obtenção relacionadas a desconhecimento ou recusa do informante, deficiências nas definições de algumas variáveis ou nas suas formas de preenchimento, maior interesse em algumas variáveis em detrimento de outras e falta de comprometimento e/ou de atenção do profissional responsável pelo preenchimento das DNVs ${ }^{2,4,7,17,19,21}$.Além disso, a pluralidade de responsáveis pelo preenchimento das DNVs exige conscientização e treinamento adequado desses profissionais como instrumento de melhoria da qualidade das suas informações ${ }^{21}$.

A omissão da idade gestacional deve ser motivo de preocupação, dada sua importância como indicador de saúde do recém-nascido. Nascimentos prematuros geram demandas adicionais sobre os serviços de saúde de alta complexidade, e suas complicações relacionamse a desfechos desfavoráveis na morbidade e mortalidade a curto e longo prazo ${ }^{22,23}$. Nesse sentido, alerta-se sobre a tendência crescente na prevalência de nascimento pré-termo no Brasil, assim como em vários países ${ }^{23}$. Dados nacionais recentes mostraram uma prevalência de 
$11,5 \%$ de nascimentos prematuros entre as crianças brasileiras, associada aos partos cesáreos e a patologias maternas/fetais ${ }^{22}$.

No atual estudo, foi possível confirmar os achados de outros pesquisadores relativos à baixa concordância das variáveis idade gestacional ${ }^{8,15,20,21}$ e quantidade de filhos mortos ${ }^{16,24-26}$. O nível de concordância pode estar influenciado pelas características dos instrumentos e dos profissionais encarregados pelo seu preenchimento. Informações menos consistentes são decorrentes de instrumentos que utilizam definições diferentes para uma mesma variável e de profissionais sem o treinamento adequado ${ }^{3,15}$.

Ainda, a acurácia das informações sobre a idade gestacional e a quantidade de filhos mortos pode estar comprometida como consequência das modificações na DNV original realizadas em 2011, com adequações na forma de mensuração de algumas questões, como o cálculo da idade gestacional e a referência às perdas fetais com a inclusão de abortos e óbitos fetais, o que exige treinamento para a implantação de tais adaptações $15,21,25,27$. Nesse contexto, ressalta-se que a definição da idade gestacional a partir do dia da última menstruação apresenta falhas decorrentes de variações na duração do ciclo menstrual e de dificuldades de memória ${ }^{15}$. Os problemas na qualidade da informação dessas variáveis inviabilizam seu uso em estudos epidemiológicos e como subsídio para as políticas públicas ${ }^{27}$.

Cabe ressaltar a necessidade de contextualizar os problemas encontrados considerando a influência do grau de desenvolvimento humano e tecnológico dos locais de estudo. Destarte, torna-se importante sensibilizar os gestores municipais quanto à importância do preenchimento correto das DNVs para as intervenções oportunas na área da saúde materno-infantil 2,7,28,29.

Neste estudo, não se incluíram algumas variáveis relevantes para a avaliação das condições sobre a saúde materno-infantil. Além disso, este trabalho apresentou restrição temporal a um ano e envolveu apenas crianças hospitalizadas em quatro instituições. Ainda que com limitações, possibilitou caracterizar a completude e a concordância de variáveis selecionadas das DNVs referentes a uma população diferenciada, composta por crianças menores de 1 ano que precisaram de internação hospitalar, sem registros similares na literatura consultada. A importância dos resultados obtidos deve ser ressaltada, considerando que a avaliação da qualidade das informações sobre nascidos vivos é fundamental para o planejamento da atenção à saúde materno-infantil e para a gestão de políticas públicas.

Este estudo permitiu verificar que a completude e a concordância da maioria das variáveis analisadas denotaram boa qualidade no preenchimento das DNVs. Entretanto, a idade gestacional sobressaiu por sua incompletude e baixa concordância. Além disso, os registros sobre a quantidade de filhos mortos merecem mais atenção ao destacar-se a concordância das informações apenas razoável. Confirmam-se, assim, entre crianças menores de 1 ano que precisaram de hospitalização, os achados de estudos anteriores relacionados à qualidade das informações sobre nascidos vivos no Brasil.

\section{REFERÊNCIAS}

1. Paiva NS, Coeli CM, Moreno AB, Guimarães RM, Camargo KR Jr. Sistema de informações sobre nascidos vivos: um estudo de revisão. Cien Saude Colet. 2011;16(Supl. 1):1211-20. http://dx.doi.org/10.1590/ S1413-81232011000700053. PMid:21503469.

2. Figueroa Pedraza D. Qualidade do sistema de informações sobre nascidos vivos (sinasc): análise crítica da literatura. Cien Saude Colet. 2012;17(10):2729-37. http://dx.doi.org/10.1590/S1413-81232012001000021. PMid:23099759.

3. Lima CRA, Schramm JMA, Coeli CM, Silva MEM. Revisão das dimensões de qualidade dos dados e métodos aplicados na avaliação dos sistemas de informação em saúde. Cad Saude Publica. 2009;25(10):2095-109. http://dx.doi.org/10.1590/S0102-311X2009001000002. PMid:19851611.

4. Oliveira MM, Andrade SSCA, Dimech GS, Oliveira GCG, Malta DC, Rabello DL No, et al. Avaliação do Sistema de Informações sobre Nascidos Vivos. Brasil, 2006 a 2010. Epidemiol Serv Saude. 2015;24(4):629-40. http:// dx.doi.org/10.5123/S1679-49742015000400005.

5. Figueroa Pedraza D. Sistema de Informações sobre Nascidos Vivos: uma análise da qualidade com base na literatura. Cad Saúde Colet. 2021;29(1):囚143-152. https://doi.org/10.1590/1414-462X202129010106. 
6. Rodrigues M, Bonfim C, Frias PG, Braga C, Gurgel IGD, Medeiros Z. Diferenciais na adequação das informações de eventos vitais nos municípios de Pernambuco, 2006-2008. Rev Bras Epidemiol. 2012;15(2):275-84. http://dx.doi.org/10.1590/S1415-790X2012000200005. PMid:22782093.

7. Maia LTS, Souza WV, Mendes ADCG, Silva AGSD. Uso do linkage para a melhoria da completude do SIM e do Sinasc nas capitais brasileiras. Rev Saude Publica. 2017;51:112. http://dx.doi.org/10.11606/S15188787.2017051000431. PMid:29211201.

8. Guimarães PV, Coeli CM, Cardoso RC, Medronho RA, Fonseca SC, Pinheiro RS. Confiabilidade dos dados de uma população de muito baixo peso ao nascer no Sistema de Informações sobre Nascidos Vivos. Rev Bras Epidemiol. 2012;15(4):694-704. http://dx.doi.org/10.1590/S1415-790X2012000400002. PMid:23515766.

9. Guimarães EAA, Hartz ZMA, Loyola AI Fo, Meira AJ, Luz ZMP. Avaliação da implantação do Sistema de Informação sobre Nascidos Vivos em municípios de Minas Gerais, Brasil. Cad Saude Publica. 2013;29(10):2105-18. http://dx.doi.org/10.1590/0102-311X00116312. PMid:24127104.

10. Romero $\mathrm{DE}$, Cunha CB. Evaluation of quality of epidemiological and demographic variables in the Live Births Information System, 2002. Cad Saude Publica. 2007;23(3):701-14. http://dx.doi.org/10.1590/S0102311X2007000300028. PMid:17334583.

11. Miot HA. Análise de concordância em estudos clínicos e experimentais. J Vasc Bras. 2016;15(2):89-92. http://dx.doi.org/10.1590/1677-5449.004216. PMid:29930571.

12. Shrout PE, Fleiss JL. Intraclass correlation: uses in assessing rater reliability. Psychol Bull. 1979;86(2):420-8. http://dx.doi.org/10.1037/0033-2909.86.2.420. PMid:18839484.

13. Sim J, Wright CC. The kappa statistic in reliability studies: use, interpretation, and sample size requirements. Phys Ther. 2005;85(3):257-68. http://dx.doi.org/10.1093/ptj/85.3.257. PMid:15733050.

14. Marques LJP, Oliveira CM, Bonfim CV, Marques LJP, Oliveira CM, Bonfim CV. Avaliação da completude e da concordância das variáveis dos Sistemas de Informações sobre Nascidos Vivos e sobre Mortalidade no Recife-PE, 2010-2012. Epidemiol Serv Saude. 2016;25(4):849-54. http://dx.doi.org/10.5123/S167949742016000400019. PMid:27869979.

15. Szwarcwald CL, Leal MC, Esteves-Pereira AP, Almeida WS, Frias PG, Damacena GN, et al. Avaliação das informações do Sistema de Informações sobre Nascidos Vivos (SINASC), Brasil. Cad Saude Publica. 2019;35(10):e00214918. http://dx.doi.org/10.1590/0102-311x00214918. PMid:31596402.

16. Gabriel GP, Chiquetto L, Morcillo AM, Ferreira MC, Bazana IGM, Daolio LD, et al. Avaliação das informações das Declarações de Nascidos Vivos do Sistema de Informação sobre Nascidos Vivos (Sinasc) em Campinas, São Paulo, 2009. Rev Paul Pediatr. 2014;32(3):183-8. http://dx.doi.org/10.1590/0103-0582201432306. PMid:25479847.

17. Silva RS, Oliveira CM, Ferreira DKS, Bonim CV. Avaliação da completude das variáveis do Sistema de Informações sobre Nascidos Vivos - Sinasc - nos Estados da região Nordeste do Brasil, 2000 e 2009 Epidemiol Serv Saude. 2013;22(2):347-52. http://dx.doi.org/10.5123/S1679-49742013000200016.

18. Paes NA, Santos CSA. As estatísticas de nascimento e os fatores maternos e da criança nas microrregiões do Nordeste brasileiro: uma investigação usando análise fatorial. Cad Saude Publica. 2010;26(2):311-22. http://dx.doi.org/10.1590/S0102-311X2010000200010. PMid:20396846.

19. Dombrowski JG, Ataíde R, Marchesini P, Souza RM, Marinho CRF. Effectiveness of the Live Births Information System in the Far-Western Brazilian Amazon. Cien Saude Colet. 2015;20(4):1245-54. http://dx.doi. org/10.1590/1413-81232015204.00792014. PMid:25923635.

20. Mascarenhas MDM, Gomes KRO. Confiabilidade dos dados do Sistema de Informações sobre Nascidos Vivos em Teresina, Estado do Piauí, Brasil - 2002. Cien Saude Colet. 2011;16(Supl. 1):1233-9. http://dx.doi. org/10.1590/S1413-81232011000700055. PMid:21503471.

21. Agranonik M, Jung RO. Qualidade dos sistemas de informações sobre nascidos vivos e sobre mortalidade no Rio Grande do Sul, Brasil, 2000 a 2014. Cien Saude Colet. 2019;24(5):1945-58. http://dx.doi. org/10.1590/1413-81232018245.19632017. PMid:31166527.

22. Leal MC, Esteves-Pereira AP, Nakamura-Pereira M, Torres JA, Theme-Filha M, Domingues RMSM, et al. Prevalence and risk factors related to preterm birth in Brazil. Reprod Health. 2016;13(S3 Supl Supl. 3):127. http://dx.doi.org/10.1186/s12978-016-0230-0. PMid:27766978.

23. Henriques LB, Alves EB, Vieira FMSB, Cardoso BB, D’Angeles ACR, Cruz OG, et al. Acurácia da determinação da idade gestacional no Sistema de Informações sobre Nascidos Vivos (SINASC): um estudo de base populacional. Cad Saude Publica. 2019;35(3):e00098918. http://dx.doi.org/10.1590/0102-311x00098918. PMid:30970098. 
24. Barbuscia DM, Rodrigues-Júnior AL. Completude da informação nas Declarações de Nascido Vivo e nas Declarações de Óbito, neonatal precoce e fetal, da região de Ribeirão Preto, São Paulo, Brasil, 20002007. Cad Saude Publica. 2011;27(6):1192-200. http://dx.doi.org/10.1590/S0102-311X2011000600016. PMid:21710016.

25. Bonilha EA, Vico ESR, Freitas M, Barbuscia DM, Galleguillos TGB, Okamura MN, et al. Cobertura, completude e confiabilidade das informações do Sistema de Informações sobre Nascidos Vivos de maternidades da rede pública no município de São Paulo, 2011. Epidemiol Serv Saude. 2018;27(1):e201712811. http:// dx.doi.org/10.5123/S1679-49742018000100011. PMid:29451610.

26. Nunes FBBF, Prudêncio PS, Carvalho JFS, Mamede FV. Incompletude de informação de nascidos vivos em São Luís/MA no ano de 2012. J Res Fundam Care Online. 2016;8(1):3705-13.

27. Lino RRG, Fonseca SC, Kale PL, Flores PVG, Pinheiro RS, Coeli CM. Tendência da incompletude das estatísticas vitais no período neonatal, estado do Rio de Janeiro, 1999-2014. Epidemiol Serv Saude. 2019;28(2):e2018131. http://dx.doi.org/10.5123/S1679-49742019000200014. PMid:31291437.

28. Silva LP, Moreira CMM, Amorim MHC, Castro DS, Zandonade E. Avaliação da qualidade dos dados do Sistema de Informações sobre Nascidos Vivos e do Sistema de Informações sobre Mortalidade no período neonatal, Espírito Santo, Brasil, de 2007 a 2009. Cien Saude Colet. 2014;19(7):2011-20. http://dx.doi. org/10.1590/1413-81232014197.08922013. PMid:25014281.

29. Stevanato JM, Gaíva MAM, Silva AMC, Stevanato JM, Gaíva MAM, Silva AMC. Tendência da cobertura do Sistema de Informações sobre Nascidos Vivos em Mato Grosso, 2000 a 2012. Epidemiol Serv Saude. 2017;26(2):265-74. http://dx.doi.org/10.5123/S1679-49742017000200004. PMid:28492768. 